
\title{
28 Research Suare \\ The Effect of Ofloxacin in Men With Elevated Prostate Specific Antigen
}

\section{Seyedhossein Rabani}

Yasuj University of Medical Sciences

\section{Ali Mousavizadeh}

Yasuj University of Medical Sciences

Seyed Mohammadreza Rabani ( $\nabla$ smrrabani@gmail.com )

Yasuj University of Medical Sciences

\section{Research Article}

Keywords: Biopsy, Ofloxacin, Prostate Cancer, Prostate Specific Antigen

Posted Date: April 30th, 2021

DOI: https://doi.org/10.21203/rs.3.rs-406257/v1

License: (1) This work is licensed under a Creative Commons Attribution 4.0 International License. Read Full License 


\section{Abstract}

Background: Antibiotic prescription is a common practice in men with elevated serum prostate specific antigen. The thought is that if patients were to have a drop in, or normalization of their PSA, we can be able to avoid unnecessary prostate biopsy. The aim of this study was to evaluate the effect of ofloxacin in men with elevated PSA.

Methods: 224 men with elevated PSA from the base of $4 \mathrm{ng} / \mathrm{ml}$ were enrolled in this study. Exclusion criteria were age less than 50 or greater than 75 , history of allergy to fluoroquinolones, cases with history of recent prostate manipulation, men on 5 alpha reductase inhibitors, and known cases of prostate cancer. After a duration of 10 days ofloxacin $200 \mathrm{mg}$ twice a day orally, PSA level was rechecked.

Results: Mean age was 61.18 year. Mean PSA level before administering antibiotic was $26.3 \mathrm{ng} / \mathrm{ml}(-21.9$ +97.4). In 120 patients (53.57\%) a significant drop in serum PSA was detected so prostate biopsy was not done and in the remainder 104 patients (46.43\%), Prostate biopsy was done that showed 65 adenocarcinomas of prostate and 39 benign prostate hyperplasia.

Conclusions: This study showed in a patient with active urinalysis (pyuria) and normal rectal examination, trans rectal ultrasonography and prostate biopsy should be post ponded and antibiotic be started. If significant drop in serum PSA was seen, antibiotic should be continued to avoid unnecessary biopsies, otherwise, no benefit in asymptomatic men with normal urinalysis, but elevated PSA.

\section{Background}

Prostate cancer $(\mathrm{PCa})$ is one of the most common visceral cancers in men. It has several risk factors including presence of androgen, advanced age, a positive family of this cancer in close relatives, and African ancestry (1). Not only it is the most common visceral cancer occurring in men, but also it has been introduced as the second most common of all diagnosed malignancies and is the sixth leading cause for cancer death globally (2). A serine protease by the name of prostate specific antigen(PSA), that its physiologic role is liquefying the seminal fluid, may be raised in PCa due to release into the circulation. This increase in PSA level may also be seen in benign prostate diseases and isn't pathognomonic for PCa. As a cancer biomarker, PSA has a widespread use both in initial diagnosis and monitoring of response to treatment. As PSA was more widely used for prostate cancer screening, increasing controversies were also encountered. A group of researchers believe that screening of CaP by using PAS, has led to diagnosis and treatment of a group of prostate cancers that pose no real threat for these patients while treatment of these patients is not accompanied with an important reduce in prostate cancer mortality. There are also controversies over the cutoff point level for PSA that should indicate prostate biopsy. On the other hand, there are many clinical and experimental studies that suggest a relationship between acute and chronic prostatitis and increased serum PSA levels $(3,4,5,6$, and7). Although this biomarker is widely used for screening and early detection of Cap, false positive screening results are not uncommon $(8,9)$. 
The effect of antibiotics in patients with leukocytosis in expressed prostate secretion (EPS), in patients with asymptomatic prostatitis, but proven histologic evidence for prostatitis, and also presence of white blood cells(WBC) in post-prostate massage voided urine (VB3), has been established (10). Many urologists prescribe antibiotic to the patients with elevated PSA and thought is that if patients were to have a drop in, or normalization of their PSA, we can be able to avoid unnecessary prostate biopsy. PSA is almost uniquely produced by the epithelial tissue of prostate, but elevated PSA in the circulation may suggests increased prostatic cellular activity, as a consequence of $\mathrm{PCa}$, but are more commonly caused by non-cancerous situations such as ethnicity, advanced age, infection, inflammation, benign prostatic hyperplasia (BPH), and even recent physical activity $(11,12)$.

Regarding the frequency of $\mathrm{PCa}$, prevention and early detection of this cancer has a major public health importance. The PSA test is widely used for screening of $\mathrm{PCa}$, but for several reasons this application is controversial $(13,14,15,16$, and 17$)$.

There are many other new biomarkers witch may improve risk stratification both in diagnosing PCa and treating it, but some of them are still considered experimental, and their efficacy have not been confirmed (18).

The aim of this study was to evaluate the effect of Ofloxacin in the treatment of patients with elevated PSA with regard to reducing PSA levels and differentiation between PCa and other causes of PSA elevation, to avoid unnecessary prostate biopsy.

\section{Method}

Two hundred twenty-four men with elevated PSA to a range of more than $4 \mathrm{ng} / \mathrm{dl}$, with negative rectal examination for cancer and candidate to trans rectal ultrasound guide prostate biopsy, were enrolled in our study.

Exclusion criteria were age less than 50 and more than 75 years, history of allergy to fluoroquinolones, cases with history of recent prostate manipulation, cases with hard nodule on rectal examination, cases that were on alpha reductase inhibitors, and known cases of prostate cancer. The patients should have at least one elevated PSA detection from the cutoff point of 4 nanogram $/ \mathrm{ml}$. All patients were submitted to physical examination and rectal exam before biopsy. Written informed consent was obtained from all patients. Ofloxacin ( $200 \mathrm{mg}$ b.i.d.) was given orally for 10 days. PSA determination was repeated 10 days after therapy. If the repeated PSA has fallen to at least $50 \%$ of the original PSA or to a level of less than 4 $\mathrm{ng} / \mathrm{ml}$, Ofloxacin by the same dose was continued for at least 20 other days, but if the reduction In PSA was less than $50 \%$ or no change, or even more elevation was seen, the next plan was trans rectal ultrasound guided prostate biopsy. The number of cores varied from 12 to 14 according to radiologist preference. Local anesthesia with trans rectal instillation of $20 \mathrm{ml}$ lidocaine $2 \%$, was performed.

Specimens were obtained and sent for histopathology report. Patients with significant PSA reduction and those with negative histopathology biopsy will be followed with PSA determination and digital rectal exam (DRE) at 6-month intervals for the first year and then yearly. All subjects completed a written 
consent after being informed about the general aspects of the work and the study was approved by the ethics yasuj university of medical sciences ethical committee on biomedical research.

\section{Result}

Mean age was 61.18 year. Mean PSA level before administering antibiotic was $26.3 \mathrm{ng} / \mathrm{ml}(-21.9+97.4)$. In 120 patients (53.57\%) a significant drop (more than $50 \%$ drop, or reaching to a level of less than 4 $\mathrm{ng} / \mathrm{ml}$ ) in serum PSA was detected. Most of these patients were those with active urinalysis, so prostate biopsy was not done in this group, and in the remainder 104 patients (46.43\%), Prostate biopsy was done that showed 65 adenocarcinomas of prostate and 39 benign prostate hyperplasia. In 114 patients (50.89\%), urine analysis showed pyuria. In comparing the baseline characteristics of the patients with and without PSA reduction after antibiotics, the first group had a significantly higher baseline PSA value (table 1 and 2).

Table 1: descriptive statistics of PSA Values in the two groups

\begin{tabular}{|c|c|c|c|c|c|c|c|c|}
\hline & \multirow[t]{2}{*}{$\mathbf{N}$} & \multirow[t]{2}{*}{ Mean } & \multirow{2}{*}{$\begin{array}{l}\text { Std. } \\
\text { Deviation }\end{array}$} & \multirow[t]{2}{*}{ Minimum } & \multirow[t]{2}{*}{ Maximum } & \multicolumn{3}{|c|}{ Percentiles } \\
\hline & & & & & & 25th & $\begin{array}{l}\text { 50th } \\
\text { (Median) }\end{array}$ & 75th \\
\hline PSA1 & 224 & 16.1444 & 22.30162 & 4.20 & 167.00 & 6.0000 & 8.8000 & 15.9500 \\
\hline PSA2 & 224 & 7.2346 & 13.73288 & 0.00 & 120.00 & 1.9700 & 3.7000 & 6.8000 \\
\hline
\end{tabular}

As the measures of PSA1(before ofloxacin administration) and PSA 2(after ofloxacin administration) Values Were non normal distribution, the mean rank of values were compared with non-parametric Wilcoxon Signed Ranks Test, that showed the mean of PSA in group 2 was less than PSA in Group 1.

Table 2: the results of comparison of mean rank of PSA between groups 1 and 2 .

\begin{tabular}{|c|c|c|c|c|}
\hline & & $\mathrm{N}$ & Mean Rank & Sum of Ranks \\
\hline \multirow[t]{4}{*}{ PSA2 - PSA1 } & Negative Ranks & $185^{\mathrm{a}}$ & 119.64 & 21535.50 \\
\hline & Positive Ranks & $38^{\mathrm{b}}$ & 48.13 & 1684.50 \\
\hline & Ties & $1^{\mathrm{c}}$ & & \\
\hline & Total & 224 & & \\
\hline a. PSA2 < PSA1 & & & Z TEST & $-10.869^{b}$ \\
\hline b. PSA2 > PSA1 & & & PVALUIE (2-tailed) & 0.000 \\
\hline c. $\mathrm{PSA} 2=\mathrm{PSA} 1$ & & & & \\
\hline
\end{tabular}


According to the results of Wilcoxon Signed Ranks Test, the mean rank of values shows significant difference. $(w=-10.87, p$-value $=0.000)$, And this means that the overall value of intervention was significant.

\section{Discussion}

Prostate specific antigen is widely used for prostate cancer (Cap) screening and early detection of Cap, but false positive screening results are not uncommon. Bacterial infections of the prostate, any manipulation of the prostate and even BPH may cause PSA to be elevated in serum. It has been suggested that in men with normal PSA after a course of antibiotic therapy, the decision for prostate biopsy could be postponed or avoided until another PSA increase be encountered $(19,20)$.

An important aim in the management of men suspected for prostate cancer is minimizing negative prostate biopsies. Nadler et al. showed the presence of chronic inflammatory cells in $64.3 \%$ of men who underwent TRUS biopsy for elevated PSA (21). Brett et al. in a group of 300 randomly selected men, found about $32 \%$ category IV prostatitis (22). In their study, serum PSA level also was significantly higher $(P=0.0004)$ than in men without prostatitis.

Although the exact implication of this finding is not completely understood, the higher PSA values could be secondary to this fact that small inflammatory foci in the prostate could alter prostate integrity more extensively than small carcinomatous foci for increasing the serum PSA level.

The present study was done to investigate whether Ofloxacin $200 \mathrm{mg}$ bid, for 10 consequent days, lowers PSA to normal levels. The clinical behavior of the pathological disease of $\mathrm{CaP}$ in its very early stages is variable and uncertain. A better understanding of CaP biology, progression and its metastatic behavior are needed to be able for diagnosing, treating, or better preventing this disease.

In our study, a significant drop in PSA ( $>50 \%$ ) or normalization of PSA was detected in 120 patients (53.57\%) after Ofloxacin prescription and most of these patients were those with active urinalysis, so in this group of the patients, prostate biopsy was not done. In the remainder 104 patients (46.43\%), Prostate biopsy was done that showed 65 adenocarcinoma of prostate (CaP) and 39 benign prostate hyperplasia (BPH). In 114 patients (50.89\%), urine analysis showed pyuria. Overall we had 65 prostate adenocarcinomas out of 224 patients participated in our study (29\%),

But it is obvious that a long term follow-up is necessary to determine if any of other men will have prostate PCa in the near future, and also larger studies are necessary to identify the optimal PSA reduction level to be valuable for post pending biopsy of prostate. Several investigations showed that

in some patients, serum PSA would have dropped even without antibiotic therapy owing to the spontaneous variation in serum PSA.

The results of our study are somewhat similar to those obtained by Bulbul MA et al. (23) in 48 selected patients with LUTS, normal DRE, normal urinalysis and elevated PSA, that usually will undergo prostate 
biopsy, their patients received Ciprofloxacin for a duration of 2 weeks.

They found, PSA reduction in $63 \%$ of the patients after antibacterial therapy, with PSA returning to normal values in $9 \%$ of them, so avoiding prostate biopsy. Twenty-five patients out of 48 patients (52\%) dropped their PSA (median of $42 \%$ drop), with $80 \%$ improvement in lower urinary tract symptoms, were not biopsied. $23 / 48$ patients (48\%) did not drop their PSA, with $65 \%$ improvement in lower urinary tract symptoms, were biopsied. 9/23 patients (39\%) had PCa. They concluded that almost $50 \%$ of patients with lower urinary tract symptoms, elevated PSA, and normal DRE will drop their PSA following 2 weeks of Ciprofloxacin thus by avoiding prostate biopsy. There are also many other studies that investigated the relation between PSA and inflammatory disorders of the prostate with contradictory results and even a significant portion of patients found to have an elevated PSA, will have a normal value if the test it is repeated $(24,25,26$, and 27$)$.

It is clear that normalization of the PSA level before trans rectal ultrasonography and prostate biopsy, does not rule out the presence of prostate cancer, because the level of PSA vary spontaneously, falling and rising an average of $15 \%$ from week to week (28).

A rise in the range of less than 20 to $46 \%$ from one year to the next year is more likely to be the result of natural biological variation than PCa (29), So, the Changes in the levels of PSA are not surprising, because the level of PSA in the fluid of the prostate is nearly one million-fold higher than that in serum, and leakage from acini of the prostate to surrounding capillaries can substantially affect the serum levels.

Consequently, the widespread use of antibiotics by urologists for men with a newly elevated PSA level, on the presumption that the patient has a bacterial prostatitis, seems to be irrational.

Although there is no evidence based data, but antibiotics are often given to patients with a raised antigen level based on the presumption that the raised PSA is due to bacterial infection (30). Antibiotics may affect the clinical course of bacterial prostatitis, but $90 \%$ of symptomatic prostatitis and nearly all cases of asymptomatic prostatitis are not caused by a bacterial infection (31).

We could not find any study confirming the effect of antibiotics on PSA level, included a control group, and also there have been no related randomized clinical trials to show that prescribing antibiotics are more likely to reduce PSA levels more than a placebo.

\section{Conclusion}

Except in the patients with bacterial prostatitis that is an uncommon condition, there is no evidence that antibiotic therapy can alter PSA levels. In our experience only a reduction In PSA level to a range of at least $50 \%$ of original level or normalization of the test in a patient with pyuria may allow post ponding TRUS prostate biopsy, providing an informed follow up for these patients, otherwise no benefit for antibiotic therapy in a man with elevated PSA, but normal rectal exam and without pyuria. 


\section{Abbreviations}

PSA: Prostate Specific Antigen

PCa: Prostate Cancer

TRUS: Trans Rectal Ultrasonography

EPS; Expressed Prostate Secretion

VB3: Voided Urine Post-prostate massage

BPH: Benign Prostatic Hyperplasia

\section{Declarations}

Ethical Approval and informed consent: The ethical Approval was done by yasuj university of medical sciences ethical committee on biomedical research and the related code is: IR.YUMS.REC.1399.168

the informed, written, signed consent was taken from each patient and is available in each individual hospital record. All methods were conducted accordance with our relevant guideline and regulations

Consent of publication: According to the institution policy a written, signed consent for research and publication is taken from each patient and is available in each individual hospital record.

Data Availability: The datasets used during the current study are available from the corresponding author on reasonable request.

Funding: None Declared.

Competing interests: The authors declare that they have no competing interests.

\section{Authors' Contribution:}

Seyedhossein Rabani: Study concept and design, Analysis and interpretation of data, Drafting of the manuscript.

Ali Mousavizadeh: Study concept and design, statistical Analysis and interpretation of data.

Seyed Mohammadreza rabani: Data acquisition, Critical revision of the manuscript for important intellectual content.

Acknowledgments: The authors would like to thank all staff of the Department of urology in Beheshti Hospital for their co-operation in data collection. 


\section{References}

1. - Nair-Shalliker V, Yap S, Nunez C, Egger S, Rodger J, Patel MI, et al. Adult body size, sexual history and adolescent sexual development, may predict risk of developing prostate cancer: Results from the New South Wales Lifestyle and Evaluation of Risk Study (CLEAR). Int J Cancer. 2017;140(3):565-74.

2. - Marianna Morlando, Concetta Paola Pelullo, Gabriella Di Giuseppe, Prostate cancer screening: Knowledge, attitudes and practices in a sample of men in Italy. A survey, PLoS ONE 12(10): 1$11 ; \mathrm{e} 0186332$.

3. - Neal Jr DE, Clejan S, Sarma D, Moon TD. Prostate specific antigen and prostatitis. I. Effect of prostatitis on serum PSA in the human and nonhuman primate. Prostate 1992; 20: 105-111.

4. - Gamè X, Vincendeau S, Palascak R, Milcent S, Fournier R, Houlgatte A. Total and free serum prostate specific antigen levels during the first month of acute prostatitis. Eur Urol 2003; 43: 702705.

5. - Hasui Y, Marutsuka K, Asada Y, Ide H, Nishi S, Osada Y. Relationship between serum prostatespecific antigen and histological prostatitis in patients with benign prostatic hyperplasia. Prostate 1994; 25: 91-96.

6. - Potts JM. Prospective identification of National Institutes of Health category IV prostatitis in men with elevated prostate specific antigen. J Urol 2000; 164: 1550-1553.

7. - Irani J, Levillain P, Goujon JM, Bon D, Dore B, Aubert J. Inflammation in benign prostatic hyperplasia: correlation with prostate specific antigen value. J Urol 1997; 157: 1301-1303.

8. - Peter T Scardino; The responsible use of antibiotics for an elevated PSA level; Nature Clinical Practice Urology (2007) 4, 1

9. - Bulbul MA1, Wazzan W, Hijaz A, Shaar A; The effect of antibiotics on elevated serum prostate specific antigen in patients with urinary symptoms and negative digital rectal examination: a pilot study; J Med Liban. 2002 Jan-Apr;50(1-2):23-5.

10. - Karel T. Buddingh, MD1; Marlies G.F. Maatje, MD2; Hein Putter, MD1; René F. Kropman, MD2; Rob C.M. Pelger, MD1. Do antibiotics decrease prostate-specific antigen levels and reduce the need for prostate biopsy in type IV prostatitis? A systematic literature review,Can Urol Assoc J 2018;12(1):E2530 .

11. - Nair-Shalliker V, Smith DP, Clements M, Naganathan V, Litchfield M, Waite L, et al. The relationship between solar UV exposure, serum vitamin D levels and serum prostate-specific antigen levels, in men from New South Wales, Australia: the CHAMP study. World J Urol. 2014;32(5):1251-7.

12. - Polascik TJ, Oesterling JE, Partin AW. Prostate specific antigen: a decade of discovery-what we have learned and where we are going. The Journal of urology. 1999;162(2):293-306. Epub 1999

13. - AIRTUM Working Group. Italian Cancer figures report; 2016. http://www.registritumori.it/PDF/AIOM2016/_numeri_del_cancro_2016.pdf.

14. - Concato J, Wells CK, Horwitz RI, Penson D, Fincke G, Berlowitz DR, et al. The effectiveness of screening for prostate cancer: a nested case-control study. Arch Intern Med 2006; 166: $38 \pm 43$. 
15. - Bartsch G, Horninger W, Klocker H, Pelzer A, Bektic J, Oberaigner W, et al.; Tyrol Prostate Cancer Screening Group. Tyrol Prostate Cancer Demonstration Project: early detection, treatment, outcome, incidence and mortality. BJU Int 2008; 101: 809-16.

16. - Jemal A, Center MM, DeSantis C, Ward EM.; Global patterns of cancer incidence and mortality rates and trends.; Cancer Epidemiol Biomarkers Prev 2010; 19: $1893 \pm 907$.

17. - Bowen DJ, Hannon PA, Harris JR, Martin DP.; Prostate cancer screening and informed decisionmaking: provider and patient perspectives; Prostate Cancer Prostatic Dis 2011; 14: 155-61.

18. - Amihay Nevo - Anojan Navaratnam• Paul Andrews; Prostate cancer and the role of biomarkers; Abdominal Radiology; 2019;https://doi.org/10.1007/s00261-019-02305-8

19. - Hochreiter W, Wolfensberger P, Danuser H, Studer UE. Antibiotic treatment of asymptomatic inflammatory prostatitis in patients with elevated psa: can biopsies be avoided? Eur Urol 2004; 3 (Suppl 2): 204; Abstract 806.

20. - Singh R, Cahill D, Popert R, O'Brien TS. Repeating the measurement of prostate-specific antigen in symptomatic men can avoid unnecessary prostatic biopsy. BJU Int 2003; 92: 932-935.

21. - Nadler RB, Humphrey PA, Smith DS, Catalona WJ, Ratliff TL. Effect of inflammation and benign prostatic hyperplasia on elevated serum prostate specific antigen levels. J Urol 1995; 154: 407-409.

22. - Brett S, Carver BS, Bozeman CB, Williams BJ, Venable DD. The prevalence of men with National Institutes of Health category IV prostatitis and association with serum prostate specific antigen. J Urol 2003; 169: 589-591.

23. - Bulbul MA, Wazzan W, Hijaz A, Shaar A.; The effect of antibiotics on elevated serum prostate specific antigen in patients with urinary symptoms and negative digital rectal examination: a pilot study. J Med Liban. 2002;50(1-2):23-5.

24. - Eastham JA et al. Polyp prevention trial study group. JAMA 2003; 289: 2695-2700.

25. - Serretta V., Catanese A, Daricello G, Liotta R, Allegro R, Martorana A, Aragona F \& Melloni D; PSA reduction (after antibiotics) permits to avoid or postpone prostate biopsy in selected patients; Prostate Cancer and Prostatic Diseases; 2008;11, 148-152.

26. - J L Boddy, D J Pike, S Al-Hayek, N Shaida \& P R Malone; An elevated PSA, which normalizes, does not exclude the presence of prostate cancer; Prostate Cancer and Prostatic Diseases; 2005; 8:349352.

27. - J Constantinou and MR Feneley; PSA testing: an evolving relationship with prostate cancer screening; Prostate Cancer and Prostatic Diseases; 2006; 9: 6-13.

28. - Peter T Scardino; The responsible use of antibiotics for an elevated PSA level; Nature Clinical Practice Urology; 2007; 4(1): doi:10.1038/ncpuro0702

29. - Ornstein DK et al. the responsible use of antibioticsfor an elevated PSA level; J Urol, 1997; 157: 2197-2182.

30. - Kaygisiz O. et al. Effects of antibacterial therapy on PSA change in the presence and absence of prostatic inflammation in patients with PSA levels between 4 and $10 \mathrm{ng} / \mathrm{ml}$; Prostate Cancer and 
Prostatic Diseases; 2006, 9: 235-238.

31. - Habermacher GM et al. prostatitis/chronic pelvic pain syndrome; Annu. Rev.Med. 2006; 57: 195206) 